\section{LESSON 12}

MARGINS:

Left, Pica 20; Elite 30;

Right, moved out of the way.

PAPER: Against the paper guide at 0 on the scale on the left. Turn up 7 single lines.

Check the 'ready-to-type' position: feet, body, fingers and eyes.

\section{Warm up}

Bounce your right thumb off the space bar.

Keep your eyes on the copy.

Tap the keys sharply.

Return the carriage smartly.

\section{Right shift key}

Keep the Ik fingers in position as well if possible.

Tap the keys sharply.

Keep your eyes on the copy.

\section{The colon (:) key}

Use the left shift key and the finger.

\section{2 key}

Practise the reach until you can type $z$ with the a finger without looking down.

Keep the fds fingers in position as you type $z$.

Keep your eyes on the copy.

Tap the keys sharply.

\section{Consolidation}

Check the 'ready-to-type' position. Return the carriage smartly.

Tap the keys sharply.

Try to type each line in $1 / 2$ minute.

Centre and lock the carriage.

Cover the machine.

\section{UNIT 3}

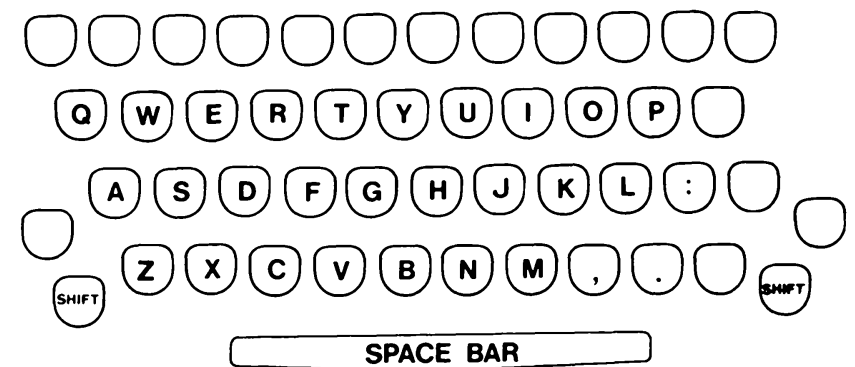

Remember to leave a blank line between sections.

Type the lines indicated by your teacher 3 times each.

1. $a b c d e f g h i j k l m n \circ p q r s t u v w x y$.

2. Under the desk they could see the extra large bin.

3. Play was held up for quite a long time after rain.

4. Once the going became easy they could catch us up.

5. Making it go was quite enough work for one person.

Type this line as many times as your teacher asks. Keeping the $j$ finger in position, depress the right shift key with the little finger of the right hand while the left hand types the required capital letter. Return little finger to ; key.

6. At; By; Cot; Do; Egg; For; Go; Ada; Dad; Fay; Fair Type the lines indicated by your teacher 3 times each.

7. Dear Sir, Dear Sir, Royal, Royal, Wales, Scotland.

8. Go. Run there. True. Say so. Do it now. I am.

9. We saw Fay, Paul, Rita, Jack, Eric and Yvonne run.

Type each line 3 times. Leave one space after a colon.

10. Day: Monday; month: January; time: half past four. 11. Shoes: type nine; colour: black; style: Cambridge.

Type this line as many times as your teacher asks.

12. aaa aza zzz azza zaz aza azf azf zaz zzz fazz fazz Type the lines indicated by your teacher 3 times each.

13. zoo zoo ze zeal zi zipp ze zest az aiz maize maize 14. Zap zoomed and zipped a maize above the jazz zeal.

15. Zed dozed a dozen times at the zoo and was frozen.

16. The zany Zulu had a dozen crazy jazz shirts ready.

Type each line 3 times. Try to build up speed each time you repeat a line.

17. Hooray: now we can all type abcdefghijklmnopqrstuvwxyz. 18. Next type, the quick brown fox jumps over the lazy dog. 19. Only by sheer hard effort, will you improve your speed. 20. As in all things, practice makes perfect, so work hard.

The two most common sizes of type face used are called Pica and Elite.

Pica gives 10 letters to every $25 \mathrm{~mm}$. Elite gives 12 letters to every $25 \mathrm{~mm}$. Use a ruler to see how many letters to every $25 \mathrm{~mm}$ you have typed.

LESSON 12 